\title{
Estimación Probabilística de Áreas de Emisión de Gases con un Robot Móvil Mediante la Integración Temporal de Observaciones de Gas y Viento
}

\author{
Carlos Sanchez-Garrido, Javier Monroy, Javier Gonzalez-Jimenez \\ Departamento de Ingeniería de Sistemas y Automática, Instituto de Investigación \\ Biomédica de Málaga, Universidad de Málaga, Campus de Teatinos, 29071, Málaga. \\ carlossanchez@uma.es, jgmonroy@uma.es, javiergonzalez@uma.es
}

\begin{abstract}
Resumen
En este trabajo se aborda el uso de un robot móvil para determinar la posición de una fuente de gas. Específicamente, nos centramos en el caso de entornos complejos, donde la presencia de múltiples habitaciones y obstáculos favorecen una dispersión caótica de los gases, lo cual dificulta en gran medida un modelado matemático del problema. Bajo estas condiciones, proponemos dividir la tarea de localización en dos fases: primero, determinar la zona del entorno donde se encuentra la fuente, y luego, determinar con precisión la localización de la misma dentro de la zona seleccionada. Centrándonos en la primera fase, proponemos el uso de un novedoso enfoque basado en un marco Bayesiano-probabilístico. Nuestro planteamiento se basa en la generación previa de un conjunto de mapas de dispersión de gas (empleando herramientas de simulación), atendiendo a las diferentes zonas del entorno y condiciones de viento. Comparando estos mapas con la información sensorial adquirida por el robot en cada instante de tiempo, derivamos la probabilidad de localización de la fuente, y guiamos el movimiento del robot hacia la dirección de máxima probabilidad. Para validar nuestro enfoque presentamos un total de 36 experimentos en los que un robot móvil localiza una fuente de gas en un entorno compuesto por múltiples habitaciones conexas.
\end{abstract}

Palabras clave: localización de fuentes de gas, robots olfativos, entornos complejos, obstáculos.

\section{Introducción}

Una fuente de gas es cualquier elemento del entorno con la propiedad de generar o liberar sustancias volátiles (gases). Esta definición abarca a una gran cantidad de objetos: recipientes abiertos, fugas en tuberías, explosivos, materia orgánica en descomposición, etc. Los gases emanados por estas fuentes se dispersan en el entorno por efecto de diferentes principios físicos, principalmente difusión y advección [2, que a su vez dependen de muchos factores ambientales: dirección y velocidad del viento, temperatura, humedad, etc. Este

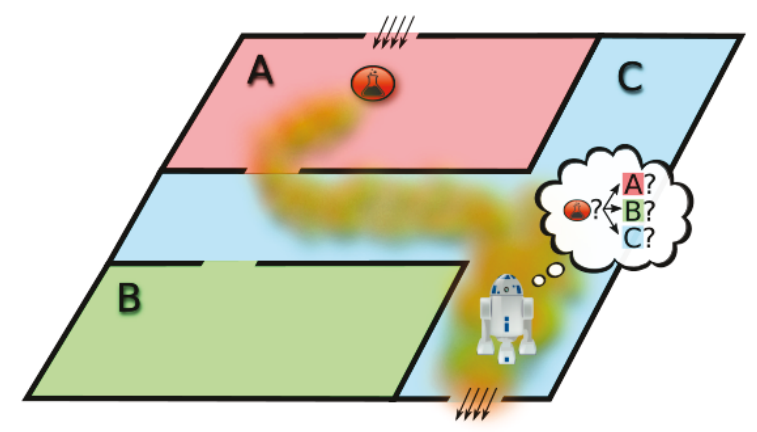

Figura 1: Búsqueda por zonas de la fuente de gas.

hecho dificulta en gran medida su correcta localización, puesto que los gases detectados a grandes distancias de su origen han podido seguir trayectos muy dispares, especialmente en entornos complejos donde la presencia de obstáculos (paredes, mobiliario, etc.) provocan turbulencias que modifican en gran medida los patrones de dispersión 23.

La búsqueda y localización de una fuente de gas consiste, por tanto, en estimar, a partir de una serie de medidas sensoriales, la posición de ésta dentro del entorno. Muchas son las aplicaciones que se beneficiarían de un sistema capaz de localizar fuentes emisoras de sustancias volátiles, entre ellas: encontrar supervivientes en zonas catastróficas, detener un incendio o fuga en las etapas iniciales detectar explosivos, drogas o agentes peligrosos, o la monitorización de vertederos y almacenes químicos. Tradicionalmente, la localización de fuentes gaseosas se ha abordado desde dos perspectivas diferentes: el despliegue de redes sensoriales 24 y el uso de vehículos autónomos con capacidad olfativa [1. La primera se basa en distribuir por el entorno una red de sensores capaces de monitorizar la composición química del aire. Este enfoque presenta las desventajas de no ser escalable al aumentar las dimensiones del entorno, así como de implicar un elevado coste de instalación y mantenimiento. La segunda opción, por la que nos decantamos en este trabajo, consiste en emplear un robot móvil, dotado con la capacidad de medir la composición química del aire mediante el uso de un conjunto de sensores de gas comúnmente deno- 
minado "nariz electrónica" 22] y de un anemómetro para medir la velocidad y dirección del viento. La ventaja de emplear un robot móvil es que se puede monitorizar los gases del entorno de forma adaptativa y con un menor coste, lo cual, añadido a la posibilidad de fusionar esta información con la proveniente de otros sistemas sensoriales del robot (láseres, cámaras, etc.), lo convierten en un enfoque muy interesante y prometedor.

No obstante, la búsqueda y localización de fuentes de gas con robots móviles no es una tarea sencilla, especialmente en entornos complejos donde la presencia de obstáculos contribuye a una dispersión caótica de los gases. Esto se aprecia en el hecho de que la mayoría de los trabajos propuestos trabajan con condiciones muy simplificadas y lejos de la realidad [17, considerando viento laminar y/o homogéneo, entornos pequeños y libres de obstáculos, etc. Bajo estas condiciones, la dispersión del gas puede ser modelada matemáticamente [5], y diversos algoritmos han sido propuestos para explotar el gradiente de concentración generado [6]. Sin embargo, estas condiciones "simplificadas" no se cumplen en la mayoría de los entornos reales, lo cual limita en gran medida su aplicación práctica.

Nuestro interés reside en la búsqueda de fuentes emisoras de gas en entornos reales, con presencia de múltiples habitaciones y obstáculos, y donde un modelado matemático de la pluma no es aplicable por la gran complejidad inherente. Bajo estas condiciones, proponemos dividir el problema de localización en dos fases, siguiendo un diseño "top-down": primero, determinar la zona del entorno donde se encuentra la fuente (véase Figura 1), y luego, determinar con precisión la localización de la misma dentro de la zona seleccionada (siendo este un espacio más simple). En este trabajo consideramos zona de emisión como el espacio conectado que abarca todas las localizaciones del entorno desde las cuales una fuente de gas genera mapas de concentración muy similares. Esto ocurre, típicamente, en habitaciones, aunque cabe mencionar que cuando estas contienen obstáculos significativos, diversas zonas de emisión pueden ser definidas.

En este trabajo nos centramos en la primera fase de la búsqueda, proponiendo un novedoso enfoque basado en un marco Bayesiano-probabilístico. Nuestro planteamiento se basa en la generación previa de un conjunto de mapas de dispersión de gas (mediante herramientas de simulación) atendiendo a las diferentes zonas del entorno y condiciones de viento. Comparando estos mapas con la información sensorial adquirida por el robot (en este trabajo consideramos localización, concentración de gas y vector de viento), ponderamos proba- bilísticamente los distintos escenarios simulados, y guiamos el movimiento del robot hacia la dirección de máxima probabilidad. Este algoritmo, además de contribuir ampliando la búsqueda de fuentes de gas a entornos complejos, combina cualquier tipo de información que exista en el entorno sobre la localización de la fuente, no sólo la obtenida a través del gas y el viento como se muestra en este trabajo, sino también otras menos utilizadas como podrían ser la visión, o la información semántica 20. Finalmente, en este trabajo nos centramos en robots móviles que se mueven en entornos planos, lo cual permite simplificar el problema a una búsqueda en dos dimensiones.

Tras un resumen de los trabajos relacionados en la sección 2 , se presenta una descripción del problema, sección 3, y la generación de los mapas de dispersión por simulación en la sección 4. El apartado 5 describe el método de localización de la fuente de gas. A continuación la sección 6 describe los experimentos y los resultados obtenidos. Concluimos con un resumen y los trabajos futuros (sección 7).

\section{Trabajos Relacionados}

El interés por la localización de fuentes de gas mediante el uso de robots dotados de capacidad olfativa no es algo novedoso y diversas estrategias han sido propuestas a lo largo de las últimas dos décadas. Los primeros trabajos, basados en el concepto de quimiotaxis o reacción de orientación como respuesta a un estímulo químico, fueron propuestos por Rozas en [19] empleando un solo robot móvil, y por Genovese 7] y Buscemi 4] para el caso de múltiples robots. Las estrategias propuestas eran en su mayoría basadas en comportamientos reactivos y bio-inspirados en animales que utilizan el sentido del olfato para realizar diversas funciones vitales. Como ejemplo, Kuwana [12] y Pyk [18] se inspiraron en como las polillas buscan a sus parejas, Grasso 8] en como las langostas buscan alimento, o Russell [21] en como localizan nutrientes las bacterias Escherichia coli.

Posteriormente, explotando el hecho de que el viento es el principal responsable en la dispersión de los gases, diferentes propuestas fueron presentadas haciendo uso, no solo de medidas sobre la concentración de los gases a estudiar, más del flujo de aire (dirección y magnitud del viento). Estas estrategias reciben el nombre de fluxotaxis (orientación en respuesta al flujo de gas). Para implementar este tipo de búsqueda con robots se utiliza la información obtenida por un anemómetro (dirección y fuerza del viento) para rastrear la pluma en dirección contraria al viento hasta llegar a la fuente emisora, como en [10] o [13]. 
En la última década, algoritmos de tipo cognitivo han ganado importancia en la comunidad científica. Por ejemplo, para minimizar la entropía de la información que se tiene sobre la posición de la fuente, [25], acuña el término infotaxis para un algoritmo que propone moverse en dirección a los lugares donde obtener mayor información sobre la localización de la fuente y no directamente a esta. Otro tipo de algoritmos utilizados son los basados en maximizar la esperanza. Estos buscan estimadores de máxima verosimilitud para determinar los parámetros de un modelo probabilístico basado en variables no observables [26].

No obstante, la mayoría de estos trabajos han sido diseñados y validados para trabajar en entornos muy simples (generalmente una habitación libre de obstáculos con viento laminar), lo cual limita su aplicabilidad a entornos reales. Una excepción son los algoritmos basados en el modelado probabilístico de la distribución del gas [15] 3], los cuales determinan la localización de la fuente tras generar un mapa de la dispersión del gas en el entorno sin asumir ningún modelo. La principal limitación de estos algoritmos es su baja eficiencia temporal, necesitando muchas observaciones distribuidas a lo largo del entorno para poder estimar correctamente la localización de la fuente.

Para una revisión más detallada de las diferentes estrategias de búsqueda de fuentes de gas, el lector puede dirigirse a trabajos como [1], [9] о [1].

\section{Definición del Problema}

El problema abordado en este trabajo es determinar la localización de una fuente de gas dentro de un entorno caracterizado por la presencia de múltiples habitaciones y obstáculos con la ayuda de un robot móvil dotado de capacidad olfativa. Para ello suponemos que la dispersión del gas se encuentra en un régimen permanente, esto es, que la fuente de gas lleve activa el suficiente periodo de tiempo como para asegurar que la dispersión del gas en el entorno ha alcanzado un estado estacionario. Además asumimos que dicho entorno se divide en $N_{r}$ posibles zonas de emisión, $\mathbf{r}=\left\{r_{i}\right\}_{i=1}^{N_{r}}$, típicamente habitaciones, siendo el objetivo determinar cual de éstas contiene la fuente de gas (véase Fig. 1).

Dado que la dispersión de un gas está fuertemente relacionada con las condiciones de viento, definimos igualmente un conjunto de mapas de viento $\mathbf{v}=\left\{\boldsymbol{v}_{j}\right\}_{j=1}^{N_{v}}$, siendo $N_{v}$ el número de diferentes condiciones de viento a considerar en el entorno de trabajo, y $\boldsymbol{v}_{j}=\left\{\mathbf{w}_{l}\right\}_{l=1}^{N}$ una cuadrícula formada por $N$ celdas, donde cada celda especifica las componentes del vector de viento en $2 \mathrm{D} \mathbf{w}=(u, v)$.

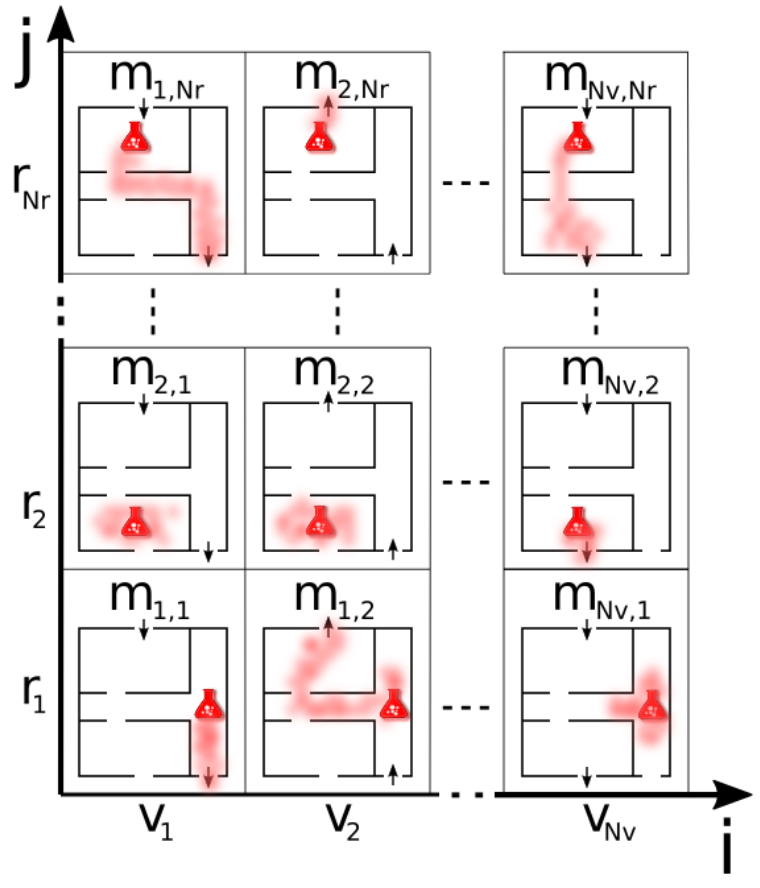

Figura 2: Mapas de distribución $(\mathbf{m})$ generados a partir de las zonas (r) y de las condiciones de viento $(\mathbf{v})$ en el entorno.

Nuestro enfoque se basa en obtener un conjunto de mapas de dispersión simulados $\mathbf{m}$, generados atendiendo a las zonas del entorno y a las diferentes condiciones de viento que se puedan dar en el mismo (véase Fig. 2), quedando definido como: $\mathbf{m}=\left\{\boldsymbol{m}_{i, j}\right\}_{i=1: N_{r}}^{j=1: N_{v}}$. Cada mapa de dispersión $\boldsymbol{m}_{i, j}$ resulta de considerar la fuente de gas en la zona $r_{i}$ bajo la influencia del flujo de viento $\boldsymbol{v}_{j}$. Como en el caso de los mapas de viento, cada $\boldsymbol{m}_{i, j}=\left\{c_{i, j}^{l}, \mathbf{w}_{i, j}^{l}\right\}_{l=1}^{N}$ se define como un mapa de cuadrícula en el que cada celda $(l)$ contiene el valor de la concentración del gas $(c)$ y del vector de viento (w) simulados.

En cada instante de tiempo $t_{k}$, el robot toma una nueva observación $\mathbf{z}_{k}=\left\{c_{k}, \mathbf{w}_{k}\right\}$ en la celda $l_{k}$, compuesta por la concentración de gas y el vector de viento en la posición actual del robot. Asumimos que ambas medidas son independientes entre si y están corrompidas por ruido Gausiano con media cero y varianza propia del sensor empleado, esto es: $\mathbf{z}_{k}=\mathcal{N}\left(\overline{\mathbf{z}}_{k}, \Sigma_{z}\right)$, donde $\overline{\mathbf{z}}_{k}=\left[\bar{c}_{k}, \bar{u}_{k}, \bar{v}_{k}\right]$ es el vector de valores medios de la observación, y la matriz de covarianza se define como:

$$
\Sigma_{z}=\left[\begin{array}{ccc}
\sigma_{c} & 0 & 0 \\
0 & \sigma_{u} & 0 \\
0 & 0 & \sigma_{v}
\end{array}\right] .
$$

Atendiendo a la relación existente entre las diversas observaciones tomadas a lo largo del tiempo, el objetivo es calcular la probabilidad de cada uno de 


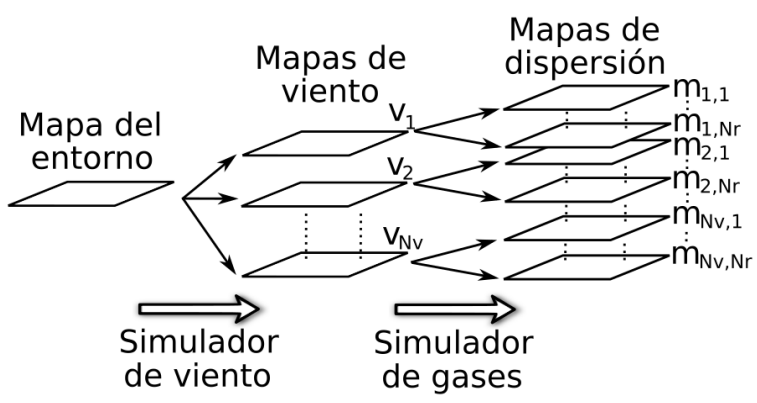

Figura 3: Fases del proceso de simulación del conjunto de mapas de dispersión m. Inicialmente se simulan $N_{v}$ mapas de viento, y luego, atendiendo a las $N_{r}$ zonas definidas en el entorno, se simulan los mapas de dispersión $\boldsymbol{m}_{n}$.

los mapas de dispersión simulados atendiendo al histórico de observaciones, esto es $p\left(\mathbf{m} \mid \mathbf{z}_{1: k}\right)$, cuyo cálculo se detalla en la Sección 5 . Finalmente, tras evaluar dicha probabilidad en cada iteración, se estima la nueva dirección de movimiento del robot hacia la zona $r_{i}$ correspondiente al mapa $\boldsymbol{m}_{i, j}$ de mayor probabilidad, o se da por terminada la búsqueda si se tiene suficiente certeza de su localización (declaración de fuente).

\section{Generación de Mapas de Dispersión}

Esta sección detalla el proceso de generación de los mapas de dispersión $\mathbf{m}$, los cuales son parte fundamental del algoritmo probabilístico propuesto en este trabajo. Dado que $\mathbf{m}$ depende del número de zonas y condiciones de viento en el entorno, el número total de mapas a considerar puede ser elevado y consecuentemente su calculo puede ser costoso. No obstante, cabe mencionar que este paso se realiza una única vez, previamente a la búsqueda de la fuente como tal (descrita en las Sección 5), por lo que el robot dispone de este conjunto de mapas desde un primer momento.

Para obtener estos mapas de dispersión proponemos usar diferentes herramientas de simulación, las cuales nos dan una aproximación sobre cómo se dispersa el gas atendiendo a los obstáculos existentes en el entorno, las condiciones de viento y la posición de la fuente (en nuestro caso la zona $r_{i}$ que contiene la fuente). Como se observa en la Fig. 3, el proceso de obtención de los mapas de dispersión se realiza en dos etapas, utilizando un simulador distinto en cada caso. En un primer paso se obtienen los datos del flujo de viento para cada una de las $N_{v}$ condiciones estudiadas, estimando en cada caso el mapa de viento $\boldsymbol{v}_{j}$ mediante el enfoque propuesto en [16], el cual aproxima el vector de viento en cada celda del entorno aten- diendo a las condiciones de contorno, la presencia de obstáculos y una serie de restricciones basadas en mecánica de fluidos. Posteriormente, estos mapas de viento son procesados por GADEN [14, un simulador de dispersión de gases que, junto a las diferentes zonas $r_{i}$ del entorno, dan lugar a los $N_{d}=N_{v} \times N_{r}$ mapas de dispersión del gas. Cada mapa generado es por tanto función de la zona $r_{i}$ donde se simula la fuente y del mapa de viento $v_{j}$ empleado durante la simulación.

\section{Método}

En este artículo proponemos un algoritmo de búsqueda que se compone de cinco fases o etapas (véase Fig. 4): toma de observaciones, pesado probabilístico, filtrado Bayesiano, evaluación y movimiento del robot. Los siguientes apartados describen en detalle cada una de estas fases.

\subsection{Pesado Probabilístico}

Para cada nueva observación $\mathbf{z}_{k}$ tomada en la celda $l_{k}$, buscamos estimar la probabilidad de cada uno de los mapas de dispersión $p\left(\boldsymbol{m}_{i, j} \mid \mathbf{z}_{k}\right)$. Para ello calculamos la distancia existente entre la observación tomada (entendida como una variable aleatoria con distribución Normal) y el valor correspondiente en esa misma celda en el mapa de dispersión, $\boldsymbol{m}_{i, j}^{l_{k}}=\left[c_{i, j}^{l_{k}}, u_{i, j}^{l_{k}}, v_{i, j}^{l_{k}}\right]$, modelado en este caso como un vector escalar, sin incertidumbre asociadd ${ }^{1}$, Esto corresponde a calcular la distancia de Mahalanobis, donde dada la naturaleza diagonal de la matriz de covarianza (véase Eq. (1)), se denomina igualmente distancia Euclídea normalizada:

$$
D M\left(\boldsymbol{m}_{i, j}^{l_{k}}, \mathbf{z}_{k}\right)=\sqrt{\left(\overline{\boldsymbol{z}}_{k}-\boldsymbol{m}_{i, j}^{l_{k}}\right)^{T} \Sigma_{z}^{-1}\left(\overline{\boldsymbol{z}}_{k}-\boldsymbol{m}_{i, j}^{l_{k}}\right)}
$$

Finalmente, la probabilidad de cada mapa de dispersión viene dada por:

$$
p\left(\boldsymbol{m}_{i, j} \mid \mathbf{z}_{k}\right)=\frac{1}{\sqrt{\left|2 \pi \Sigma_{z}\right|}} \exp \left(\frac{-D M\left(\boldsymbol{m}_{i, j}^{l_{k}}, \mathbf{z}_{k}\right)^{2}}{2}\right)
$$

\subsection{Filtrado Bayesiano}

En la Sección anterior hemos derivado la probabilidad a posteriori de cada uno de los mapas de dispersión de gas a partir de una observación puntual $\mathbf{z}_{k}$. En esta sección buscamos dar robustez al sistema, integrando la información de todas las observaciones hasta el instante de tiempo actual.

\footnotetext{
${ }^{1}$ Considerar una incertidumbre asociada a los datos simulados de los mapas de dispersión se deja para trabajo futuro.
} 


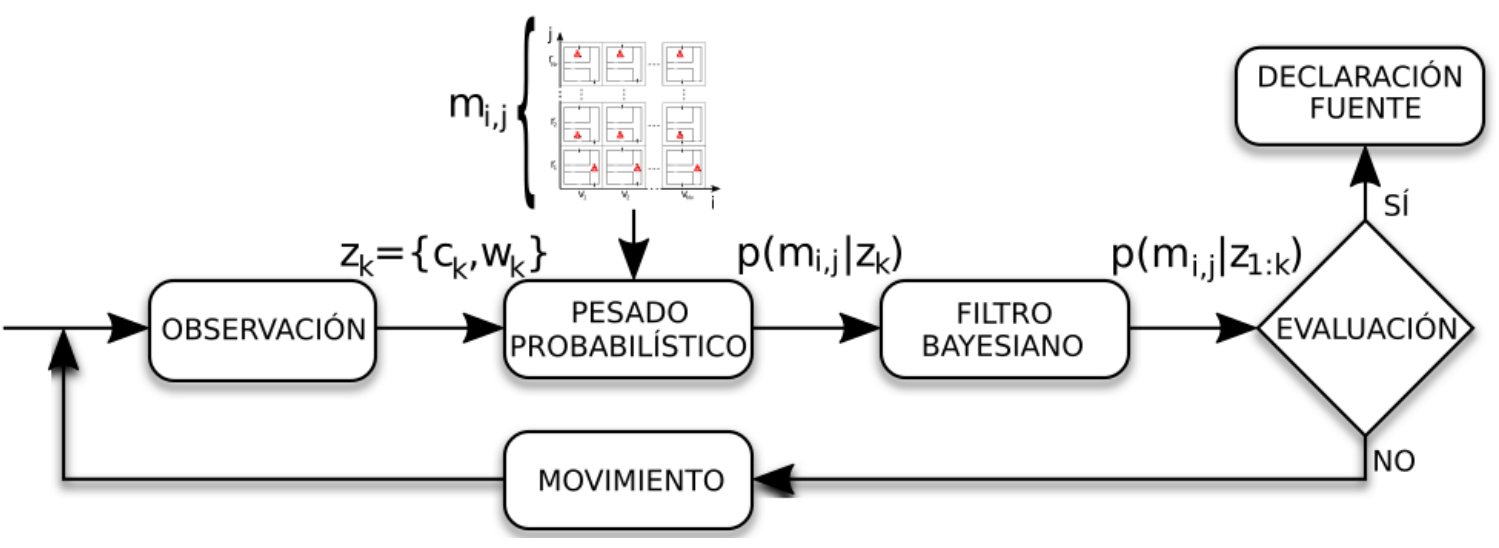

Figura 4: Diagrama del método para la localización de la zona en la que se encuentra la fuente de gas.

Para ello hacemos uso de la versión recursiva del filtro de Bayes, el cual define el belief en el instante de tiempo actual $\operatorname{Bel}_{k}\left(\boldsymbol{m}_{i, j}\right)=p\left(\boldsymbol{m}_{i, j} \mid \mathbf{z}_{1: k}\right)$ en función del belief en el instante de tiempo anterior $\operatorname{Bel}_{k-1}\left(\boldsymbol{m}_{i, j}\right)$ y la observación más reciente $\mathbf{z}_{k}$.

Para derivar la formulación específica a nuestro problema, comenzamos aplicando el teorema de Bayes al belief objetivo:

$$
\begin{aligned}
\operatorname{Bel}_{k}\left(\boldsymbol{m}_{i, j}\right) & =\frac{p\left(\mathbf{z}_{k} \mid \boldsymbol{m}_{i, j}, \mathbf{z}_{1: k-1}\right) p\left(\boldsymbol{m}_{i, j} \mid \mathbf{z}_{1: k-1}\right)}{p\left(\mathbf{z}_{k} \mid \mathbf{z}_{1: k-1}\right)} \\
& =\frac{p\left(\mathbf{z}_{k} \mid \boldsymbol{m}_{i, j}\right) p\left(\boldsymbol{m}_{i, j} \mid \mathbf{z}_{1: k-1}\right)}{p\left(\mathbf{z}_{k} \mid \mathbf{z}_{1: k-1}\right)}
\end{aligned}
$$

donde el último paso explota la suposición de Markov que especifica que el estado es completo, es decir, $p\left(\mathbf{z}_{k} \mid \boldsymbol{m}_{i, j}, \mathbf{z}_{1: k-1}\right)=p\left(\mathbf{z}_{k} \mid \boldsymbol{m}_{i, j}\right)$. Aplicando de nuevo la regla de Bayes al término $p\left(\mathbf{z}_{k} \mid \boldsymbol{m}_{i, j}\right)$ tenemos que:

$$
B e l_{k}\left(\boldsymbol{m}_{i, j}\right)=\frac{p\left(\boldsymbol{m}_{i, j} \mid \mathbf{z}_{k}\right) p\left(\mathbf{z}_{k}\right) p\left(\boldsymbol{m}_{i, j} \mid \mathbf{z}_{1: k-1}\right)}{p\left(\boldsymbol{m}_{i, j}\right) p\left(\mathbf{z}_{k} \mid \mathbf{z}_{1: k-1}\right)} .
$$

Para eliminar la dependencia de los términos que no son función de $\mathbf{m}$ aplicamos $\log$-odds, obteniendo una expresión alternativa del filtrado que simplifica el proceso de actualización al considerar nuevas observaciones:

$$
\begin{aligned}
\operatorname{lod}_{i, j}^{k}= & \log \left(\frac{B e l_{k}\left(\boldsymbol{m}_{i, j}\right)}{B e l_{k}\left(\neg \boldsymbol{m}_{i, j}\right)}\right) \\
= & \log \left(\frac{p\left(\boldsymbol{m}_{i, j} \mid \mathbf{z}_{k}\right)}{p\left(\neg \boldsymbol{m}_{i, j} \mid \mathbf{z}_{k}\right)}\right)+\log \left(\frac{p\left(\neg \boldsymbol{m}_{i, j}\right)}{p\left(\boldsymbol{m}_{i, j}\right)}\right) \\
& +\log \left(\frac{p\left(\boldsymbol{m}_{i, j} \mid \mathbf{z}_{1: k-1}\right)}{p\left(\neg \boldsymbol{m}_{i, j} \mid \mathbf{z}_{1: k-1}\right)}\right)
\end{aligned}
$$

Analizando esta ecuación observamos que el logodds de nuestro filtro se compone de tres términos: el primero hace referencia al posterior dada la observación actual, cuyo cálculo se detalla en la Sección 5.1. El segundo termino es el prior del $\log$-odds $\left(\operatorname{lod}_{i, j}^{0}\right)$. En este trabajo suponemos que no existe conocimiento a priori sobre la localización de la fuente, por lo que todos los mapas de dispersión son igualmente probables, o lo que es lo mismo $p\left(\boldsymbol{m}_{i, j}\right)=1 / N_{d}$. Finalmente, el último término hace referencia al log-odds del belief en el instante $k-1$, lo cual nos permite obtener la formula recursiva del filtro:

$$
\operatorname{lod}_{i, j}^{k}=\log \left(\frac{p\left(\boldsymbol{m}_{i, j} \mid \mathbf{z}_{k}\right)}{1-p\left(\boldsymbol{m}_{i, j} \mid \mathbf{z}_{k}\right)}\right)+\operatorname{lod} d_{i, j}^{0}+\operatorname{lod}_{i, j}^{k-1}
$$

Para recuperar la probabilidad de cada $\boldsymbol{m}_{i, j}$ en el instante $k$ se utiliza la siguiente relación:

$$
p\left(\boldsymbol{m}_{i, j} \mid \mathbf{z}_{1: k}\right)=1-\left(e^{l o d_{i, j}^{k}}\right)^{-1}
$$

\subsection{Evaluación}

Una vez estimadas las probabilidades a posteriori de los mapas de dispersión, evaluamos si la solución obtenida converge a un caso particular $\boldsymbol{m}_{i, j}$. Para ello imponemos la condición $p\left(\boldsymbol{m}_{i, j} \mid \mathbf{z}_{1: k}\right) \geq p_{t h}$ durante un periodo de tiempo $t_{t h}$. Si dicha condición se cumple, se declara la fuente como encontrada en la zona $r_{i}$, mientras que si no se cumple, el robot selecciona la siguiente posición para moverse siguiendo la estrategia descrita en el apartado siguiente, volviendo a tomar una nueva observación.

\subsection{Estrategia de Movimiento del Robot}

Diversas son las estrategias de movimiento que pueden implementarse atendiendo a $p\left(\boldsymbol{m}_{i, j} \mid \mathbf{z}_{1: k}\right)$. En este trabajo optamos por una solución simple, proponiendo un desplazamiento del robot hacia la zona $r_{i}^{*}$ correspondiente al mapa de dispersión más probable:

$$
r_{i}^{*}=\underset{i}{\operatorname{argmáx}} p\left(\boldsymbol{m}_{i, j} \mid \mathbf{z}_{1: k}\right)
$$




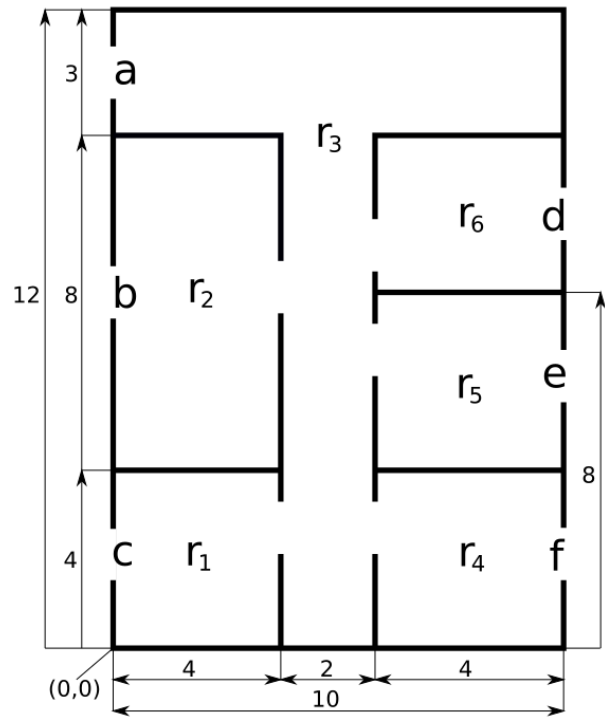

Figura 5: Entorno de prueba compuesto de seis zonas $\left(r_{1}-r_{6}\right)$ y seis aperturas al exterior $(a-f)$ (todas las distancias están expresadas en metros).

Al tratarse de un entorno con obstáculos, asumimos que el robot dispone de sistemas de navegación reactiva y de planificación de caminos, permitiéndole determinar los comandos de movimiento necesarios para alcanzar el objetivo propuesto.

\section{Experimentos y Resultados}

Con intención de verificar el funcionamiento del método propuesto, en este apartado se realizan un total de 36 experimentos en los que el objetivo es determinar la zona de emisión de la fuente en el entorno que aparece en la Fig. 5. En cada experimento, el robot parte desde la posición $(x, y)=(0,0)$ desplazándose una celda en cada instante de tiempo (siendo $10 \mathrm{~cm}$ el tamaño de los lados de la celda), en dirección a la zona de emisión $r_{i}$ correspondiente al mapa de dispersión de máxima probabilidad. Para la toma de observaciones, el robot está equipado con un sensor de gas con $\sigma_{c}=0,1 p p m$ y un anemómetro que mide el módulo y dirección del viento con $\sigma_{u}=\sigma_{v}=0,2 \mathrm{~m} / \mathrm{s}$.

Para generar el conjunto de mapas de dispersión m se ha simulado en primer lugar el conjunto de mapas de viento $\mathbf{v}$ para todos los casos donde dos de las aperturas están abiertas simultáneamente mientras que el resto permanecen cerradas, de forma que el viento (de módulo $1 \mathrm{~m} / \mathrm{s}$ en la posición de la apertura por la que entra) circula de una a otra y viceversa, obteniendo $N_{v}=30$ condiciones de viento. A continuación, se han realizado las simulaciones de dispersión de gas, situando la fuente (que libera gas a razón de $10 \mathrm{ppm} / \mathrm{s}$ ) en cada una de las $N_{r}=6$ zonas, para cada uno de los mapas de viento, obteniendo un total de $N_{d}=180$ mapas de dispersión.

A partir de esta configuración, se ha generado un nuevo conjunto de 36 mapas de dispersión (que no forman parte de $\mathbf{m}$ ), para validar el funcionamiento del método propuesto. Estos mapas han sido generados de forma que la dispersión del gas afecta a gran parte del entorno (es decir, colocando la fuente de gas en el flujo principal del viento), lo cual garantiza poder detectar la emisión de gas desde la distancia y posibilitar su localización.

\subsection{Análisis de los resultados}

La figura 6 muestra uno de los 36 experimentos realizados, donde se puede observar como el gas emitido se dispersa por efectos del viento, afectando a diversas habitaciones del entorno (a). El resto de sub-figuras (b-e) muestra la evolución, para diversos instantes de tiempo, de la trayectoria del robot hacia la zona de máxima probabilidad y el peso que se otorga a cada uno de los mapas de dispersión $\boldsymbol{m}_{i, j}$.

Para determinar la eficiencia del método, se ha realizado una comparación del tiempo que emplea el robot en la búsqueda de la fuente para los 36 experimentos frente a una búsqueda secuencial, cuyos resultados aparecen en la figura 7. En la búsqueda secuencial el robot recorre una a una las habitaciones del entorno, dirigiéndose al centro de éstas en el siguiente orden preestablecido $\left(r_{1}, r_{4}, r_{5}, r_{2}, r_{6}, r_{3}\right)$. Como se observa, nuestro método reduce en la mayoría de los casos el tiempo empleado, obteniendo una mejora media del $31 \%$ con respecto a la búsqueda secuencial, acentuándose esta ganancia cuando la distancia inicial a la fuente se hace mayor.

\section{Conclusiones y trabajos futuros}

En este trabajo se ha abordado el problema de localizar una fuente de gas en un entorno donde existen múltiples habitaciones y obstáculos que determinan el comportamiento de la dispersión de la sustancia volátil que se libera. El enfoque propuesto está basado en el uso de un robot móvil con capacidad olfativa, el cual estima la localización de la fuente en cada instante de tiempo atendiendo a una seria de medidas sensoriales (gas y viento). Concretamente, hemos presentado un novedoso método probabilístico basado en un filtrado Bayesiano de un conjunto de mapas de dispersión simulados, el cual determina de forma iterativa la zona de emisión de máxima probabilidad en cada instante de tiempo. Para validar el funcionamiento del algoritmo se han presentado los resultados de un conjunto de experimentos realizados en un 


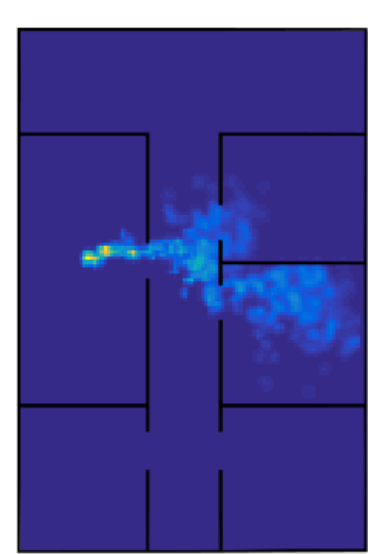

(a)

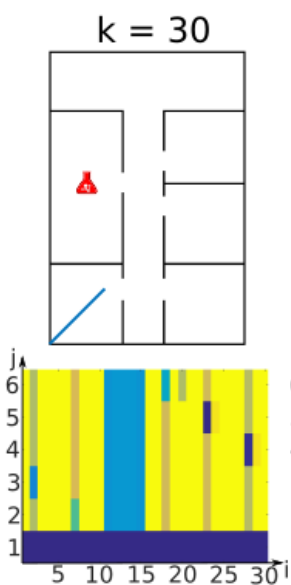

(b)

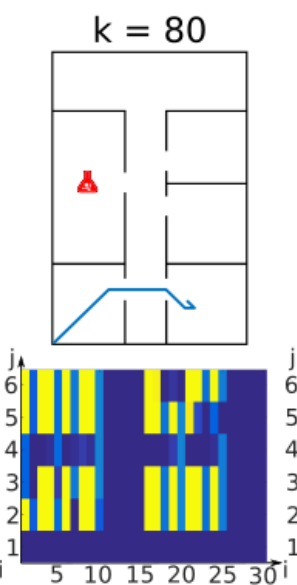

(c)

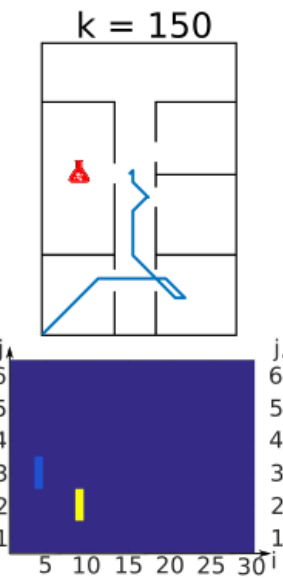

(d)

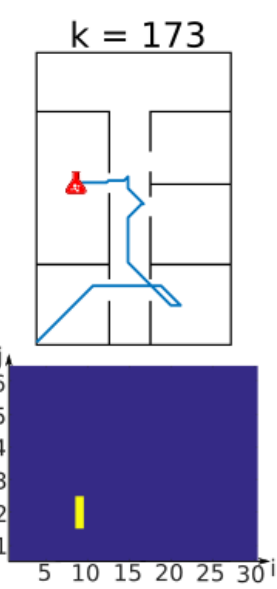

(e)

Figura 6: Resultado de la búsqueda de la zona de emisión de la fuente para una de las simulaciones. El mapa en (a) muestra la concentración de gas existente en el entorno. El subconjunto de mapas (b-e) ilustra la trayectoria del robot en los instantes de tiempo k y el nivel de creencia para el conjunto $\mathbf{m}$ de mapas de dispersión (en amarillo los mapas para los que el robot determina una mayor creencia y en azul para los descartados.

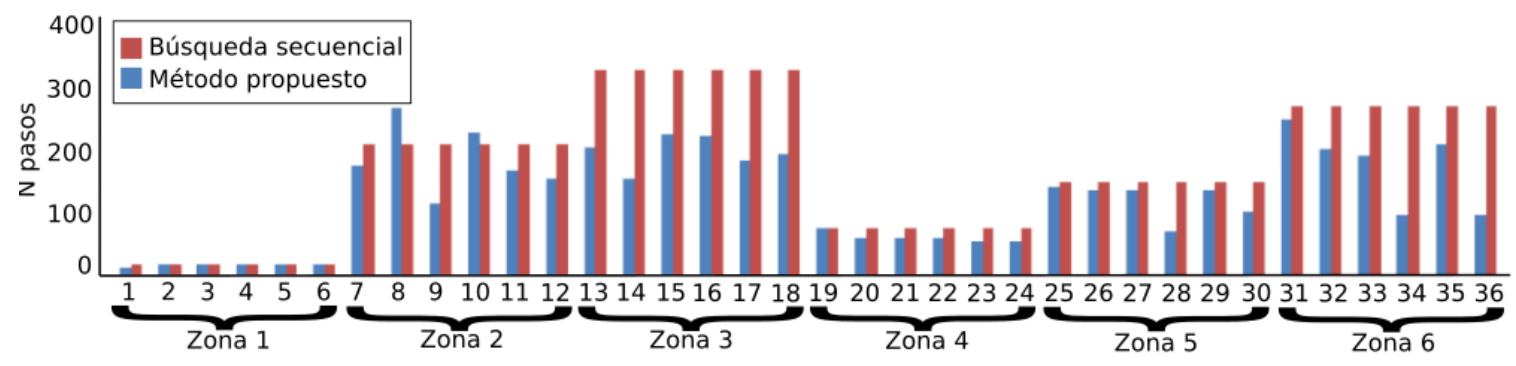

Figura 7: Comparación del número de pasos para los 36 experimentos de búsqueda de la zona de emisión de la fuente de gas considerados en este trabajo. Las llaves inferiores indican la zona en la que se encuentra la fuente en cada experimento.

entorno complejo formado por múltiples habitaciones conexas. Estos resultados han demostrado la aplicabilidad del método propuesto para la localización de fuentes de gas en entornos reales, mostrando una mejora media del $31 \%$ en el tiempo de búsqueda en comparación con un algoritmo secuencial.

En próximos trabajos se estudiará el uso de otros tipos de informaciones existentes en el entorno que puedan indicar la localización de la fuente de gas, como pueden ser la visión o la información semántica. Se pretende estudiar el uso de distintas estrategias de movimiento, como moverse en la dirección de máxima información o en la dirección que minimice la entropía sobre la información de la posición de la fuente. También se abordará la segunda parte en la que se ha divido el problema de localización, estudiando distintas estrategias para declarar la posición exacta de la fuente en la zona.

\section{Agradecimientos}

Este trabajo ha sido parcialmente financiado a través del plan nacional, gobierno de España (proyecto DPI2014-55826-R) y por la junta de Andalucía (proyecto de excelencia TEP2012-530).

\section{Referencias}

[1] B. Bayat, N. Crasta, A. Crespi, A. M. Pascoal, and A. Ijspeert. Environmental monitoring using autonomous vehicles: a survey of recent searching techniques. Current Opinion in Biotechnology, 45:76-84, 2017.

[2] G. A. Bird. Molecular gas dynamics. NASA STI/Recon Technical Report A, 76, 1976.

[3] J. L. Blanco, J. G. Monroy, A. Lilienthal, and J. Gonzalez-Jimenez. A kalman filter based approach to probabilistic gas distribution mapping. In Proceedings of the 28th Annual ACM Symposium on Applied Computing, pages 217-222. ACM, 2013.

[4] L. Buscemi, M. Prati, and G. Sandini. Cellu- 
lar robotics: behaviour in polluted environments. 1994.

[5] J. A. Farrell, J. Murlis, X. Long, W. Li, and R. T. Cardé. Filament-based atmospheric dispersion model to achieve short time-scale structure of odor plumes. Environmental fluid mechanics, 2(1):143-169, 2002.

[6] J. A. Farrell, S. Pang, and W. Li. Chemical plume tracing via an autonomous underwater vehicle. IEEE Journal of Oceanic Engineering, 30(2):428442, 2005.

[7] V. Genovese, P. Dario, R. Magni, and L. Odetti. Self organizing behavior and swarm intelligence in a pack of mobile miniature robots in search of pollutants. In Intelligent Robots and Systems, 1992., Proceedings of the 1992 lEEE/RSJ International Conference on, volume 3, pages 15751582. IEEE, 1992.

[8] F. W. Grasso, J. H. Dale, T. R. Consi, D. C. Mountain, and J. Atema. Behavior of purely chemotactic robot lobster reveals different odor dispersal patterns in the jet region and the patch field of a turbulent plume. The Biological Bulletin, 191(2):312-313, 1996.

[9] M. Hutchinson, H. Oh, and W.-H. Chen. A review of source term estimation methods for atmospheric dispersion events using static or mobile sensors. Information Fusion, 36:130-148, 2017.

[10] H. Ishida, K. Hayashi, M. Takakusaki, T. Nakamoto, T. Moriizumi, and R. Kanzaki. Odoursource localization system mimicking behaviour of silkworm moth. Sensors and Actuators A: Physical, 51(2-3):225-230, 1995.

[11] G. Kowadlo and R. A. Russell. Robot odor localization: a taxonomy and survey. The International Journal of Robotics Research, 27(8):869-894, 2008.

[12] Y. Kuwana, I. Shimoyama, and H. Miura. Steering control of a mobile robot using insect antennae. In Intelligent Robots and Systems 95.'Human Robot Interaction and Cooperative Robots', Proceedings. 1995 IEEE/RSJ International Conference on, volume 2, pages 530-535. IEEE, 1995.

[13] A. J. Lilienthal, D. Reimann, and A. Zell. Gas source tracing with a mobile robot using an adapted moth strategy. In $A M S$, pages 150-160, 2003.

[14] J. Monroy, V. Hernandez-Bennets, H. Fan, A. Lilienthal, and J. Gonzalez-Jimenez. Gaden: A 3d gas dispersion simulator for mobile robot olfaction in realistic environments. Sensors, 17(7): 1479, 2017.

[15] J. G. Monroy, J.-L. Blanco, and J. G. Jiménez. Time-variant gas distribution mapping with obstacle information. Auton. Robots, 40(1):1-16, 2016.

[16] J. G. Monroy, M. Jaimez, and J. GonzalezJimenez. Online estimation of $2 \mathrm{~d}$ wind maps for olfactory robots. 2017.
[17] S. Pang and J. A. Farrell. Chemical plume source localization. IEEE Transactions on Systems, Man, and Cybernetics, Part B (Cybernetics), 36 (5):1068-1080, 2006.

[18] P. Pyk, S. Bermúdez i Badia, U. Bernardet, P. Knüsel, M. Carlsson, J. Gu, E. Chanie, B. S. Hansson, T. C. Pearce, and P. F. J. Verschure. An artificial moth: Chemical source localization using a robot based neuronal model of moth optomotor anemotactic search. Autonomous Robots, 20(3):197-213, 2006.

[19] R. Rozas, J. Morales, and D. Vega. Artificial smell detection for robotic navigation. In Advanced Robotics, 1991.'Robots in Unstructured Environments', 91 ICAR., Fifth International Conference on, pages 1730-1733. IEEE, 1991.

[20] J.-R. Ruiz-Sarmiento, C. Galindo, and J. Gonzalez-Jimenez. Building multiversal semantic maps for mobile robot operation. Knowledge-Based Systems, 119:257-272, 2017.

[21] R. A. Russell, A. Bab-Hadiashar, R. L. Shepherd, and G. G. Wallace. A comparison of reactive robot chemotaxis algorithms. Robotics and Autonomous Systems, 45(2):83-97, 2003.

[22] C. Sanchez-Garrido, J. G. Monroy, and J. Gonzalez-Jimenez. A configurable smart e-nose for spatio-temporal olfactory analysis. In SENSORS, 2014 IEEE, pages 1968-1971. IEEE, 2014.

[23] B. I. Shraiman and E. D. Siggia. Scalar turbulence. Nature, 405(6787):639-646, 2000.

[24] L. Shu, M. Mukherjee, X. Xu, K. Wang, and $\mathrm{X}$. Wu. A survey on gas leakage source detection and boundary tracking with wireless sensor networks. IEEE Access, 4:1700-1715, 2016.

[25] M. Vergassola, E. Villermaux, and B. I. Shraiman. 'infotaxis' as a strategy for searching without gradients. Nature, 445(7126):406-409, 2007.

[26] S. Vijayakumaran, Y. Levinbook, and T. F. Wong. Maximum likelihood localization of a diffusive point source using binary observations. IEEE Transactions on Signal Processing, 55(2): 665-676, 2007. 